\title{
Modulation instability-induced fading in phase-sensitive optical time-domain reflectometry
}

\author{
Hugo F. Martins, ${ }^{1,2}$ Sonia Martin-Lopez, ${ }^{3}$ Pedro Corredera, ${ }^{3}$ Pedro Salgado, ${ }^{3}$ \\ Orlando Frazão, ${ }^{1}$ and Miguel González-Herráez ${ }^{4, *}$ \\ ${ }^{1}$ INESC Porto, Rua do Campo Alegre, 687, Porto 4169-007, Portugal \\ ${ }^{2}$ Faculdade de Ciências da Universidade do Porto, Rua do Campo Alegre, 687, Porto 4169-007, Portugal \\ ${ }^{3}$ Instituto de Óptica, CSIC, C/Serrano 144, Madrid 28006, Spain \\ ${ }^{4}$ Departamento de Electrónica, Universidad de Alcalá, Escuela Politécnica DO-231, Madrid 28871 Spain \\ *Corresponding author: miguelg@depeca.uah.es
}

Received January 2, 2013; revised February 6, 2013; accepted February 7, 2013;

posted February 8, 2013 (Doc. ID 182628); published March 12, 2013

Phase-sensitive optical time-domain reflectometry ( $\phi \mathrm{OTDR})$ is a simple and effective tool allowing the distributed monitoring of vibrations along single-mode fibers. We show in this Letter that modulation instability (MI) can induce a position-dependent signal fading in long-range $\phi$ OTDR over conventional optical fibers. This fading leads to a complete masking of the interference signal recorded at certain positions and therefore to a sensitivity loss at these positions. We illustrate this effect both theoretically and experimentally. While this effect is detrimental in the context of distributed vibration analysis using $\phi \mathrm{OTDR}$, we also believe that the technique provides a clear and insightful way to evidence the Fermi-Pasta-Ulam recurrence associated with the MI process. (c) 2013 Optical Society of America

OCIS codes: $\quad 290.5900,190.2640,060.2370,190.4370,190.3270$.

Phase-sensitive optical time-domain reflectometry $(\phi \mathrm{OTDR})$ is a powerful technique that allows the fully distributed monitoring of vibrations along an optical fiber cable. This technique has attracted considerable attention due to its application in the monitoring of intrusions over large perimeters. Conventional systems described in the literature allow the distributed measurement of vibrations of up to $1 \mathrm{kHz}$ with a resolution of $5 \mathrm{~m}$ and dynamic range of a few tens of kilometers $(<50 \mathrm{~km})[1,2]$.

A $\phi$ OTDR works by injecting a pulse of highly coherent light into a conventional single-mode fiber. Unlike traditional OTDRs, which can only measure intensity variations along the fiber, in a $\phi \mathrm{OTDR}$ the light reflected from different scattering centers interferes coherently to produce the detected optical power trace. The detected value at a certain position is therefore sensitive to the relative phases among the reflected fields coming from the different scattering centers around that position. In the case of localized vibrations, the trace shows variations synchronized with the vibration frequency.

To achieve reliable vibration measurements, it is indispensable to have a good signal-to-noise ratio (SNR) in the measured trace. In addition, it is generally desired to have the best possible range and resolution. The range, resolution, and SNR are tightly related parameters. For a given resolution (input pulse width), an increase of dynamic range and SNR of a $\phi$ OTDR sensor can only be achieved by increasing the input pump peak power. However, the input pump peak power cannot be indefinitely increased due to the onset of nonlinear effects. Some of these nonlinear limitations have been briefly described in the general context of coherent OTDRs [3]. Among these nonlinear limitations, the first effect to arise in usual conditions for $\phi$ OTDR is modulation instability (MI).

MI in fibers results from the interplay of the Kerr effect and anomalous dispersion. In the spectral domain, MI manifests as the buildup of two sidebands at each side of the center beam wavelength. MI has been theoretically and experimentally described $[4,5]$. In the strong conversion regime, the MI process exhibits a reversible power exchange between the pump and the sidebands known as the Fermi-Pasta-Ulam (FPU) recurrence [6]. In the specific context of distributed fiber sensors, MI has been shown to limit the performance of distributed fiber sensors based on stimulated Brillouin scattering [7]. In the specific case of $\phi \mathrm{OTDR},[3]$ provided useful limits to avoid the effect of MI in the general case of coherent OTDRs. However, in this study, there was no in-depth description of the effect of MI on the trace.

In this Letter, the authors present an in-depth experimental and theoretical description of the impact of MI in the performance of a $\phi$ OTDR. We show theoretically and experimentally that MI generally leads to a localized fading of the interference recorded in the trace and therefore to a loss of sensitivity in these positions. This fading is attributed to the reversible power transfer of the main input laser line to the MI sidebands (FPU recurrence). Although this effect can be considered detrimental in the context of $\phi \mathrm{OTDR}$, we believe that it can be a convenient and highly visual way of evidencing the FPU recurrence in optical fibers.

The experimental setup used to observe the effect of MI on $\phi$ OTDR is shown in Fig. 1. A highly coherent laser diode (LD) (linewidth of $1.6 \mathrm{MHz}$ ) emitting at $1546 \mathrm{~nm}$ was used as the light source. A semiconductor optical amplifier (SOA), with rise/fall times in the order $2.5 \mathrm{~ns}$, driven by a waveform signal generator (SG), was used to create $50 \mathrm{~ns}$ almost square pulses. A polarization controller (PC) was placed before the SOA to optimize the modulation properties and avoid any polarizationsensitivity issue. Between the signal pulses, the SOA was negatively biased so as to enhance the extinction ratio (ER) of the delivered pulses. An ER of $>50 \mathrm{~dB}$ was achieved this way. In this configuration, the ER has a very high impact in the SNR of the detected trace. In order to 
minimize the effect of the amplified spontaneous emission (ASE) added by the erbium-doped fiber amplifier (EDFA), we inserted a tunable fiber Bragg grating (FBG) working in reflection. The spectral profile is the typical spectrum of a $100 \%$ reflection FBG, and its spectral width is $0.8 \mathrm{~nm}$. Before being coupled into $10 \mathrm{~km}$ of SMF-28 fiber (FUT), light passed through an attenuator, which allowed varying the input power in the fiber. The average optical input power was measured at this position using a calibrated tap coupler (not shown in the figure), and the signal backreflected from the fiber was recorded with a $125 \mathrm{MHz}$ photodetector and a high-speed digitizer. An optical spectrum analyzer (OSA) with a resolution of $20 \mathrm{pm}$ was used to observe the signal spectrum at the end of the fiber.

$\phi$ OTDR traces and output spectra were recorded with increasing values of input pulse powers. For low input powers $(<400 \mathrm{~mW})$, the traces displayed the behavior shown in the inset of Fig. 2 (the losses along the fiber have been numerically eliminated to improve the visualization). As is visible, the trace displays the expected random oscillations, where the amplitude of the oscillations remains constant all along the fiber (the interference does not lose contrast along the propagation). With higher input powers, we could record complete fading of the trace oscillations (i.e., very low visibility) at specific positions, as shown in Fig. 2 (e.g., for $1.25 \mathrm{~W}$ pulse peak power, we could record complete signal fading at 4.4, 6.8, and $9.5 \mathrm{~km}$ ). With increasing powers, the number of fading points is increased and the position of the first fading point comes closer to the input end of the fiber. Because the sensitivity of the $\phi$ OTDR depends on the visibility of the interferences, we can conclude that in these positions we would have almost no vibration sensitivity. It is also noticeable that the overall sensitivity would also be decreasing rapidly, thus severely reducing the performance of the sensor.

These results can be interpreted as follows: for low powers $(<400 \mathrm{~mW})$, the effect of $\mathrm{MI}$ is negligible and the propagation of the pulse along the fiber can be thought of as purely linear. In these conditions, the spectral content of the pulse remains unaltered and the visibility of the interference remains constantly high along the fiber. For higher input powers, however, the MI gain is correspondingly increased. This leads to a depletion of the main laser line in favor of the sidebands. Because the sidebands are spectrally broad, the visibility of the detected interference will correspondingly decrease, and the contrast of the oscillations will diminish. However, as shown in previous works on FPU recurrence [5, $\underline{6}]$, with increasing distance or input power, this power transfer is reversed after a certain minimum point,

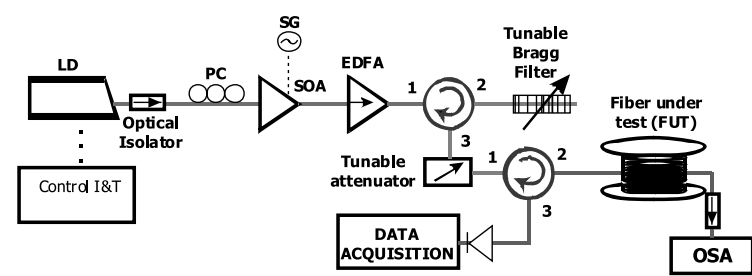

Fig. 1. Experimental setup. Acronyms are explained in the text.

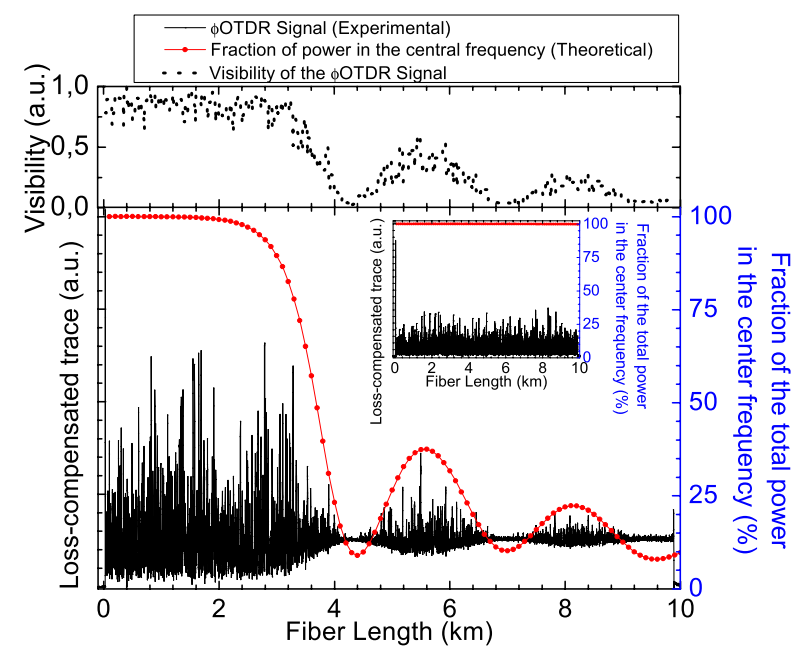

Fig. 2. (Color online) $\phi$ OTDR signal along the fiber under test (FUT) for an input pump peak power of $\sim 1.25 \mathrm{~W}$ (main figure) and $\sim 0.35 \mathrm{~W}$ (inset figure). Fiber losses have been eliminated along the trace to improve visualization. The theoretical fraction of power contained in the central wavelength is also presented in both cases. The top figure shows the visibility of the $\phi O T D R$ interference signal for the main figure signal. The visibility is computed as $V=\left(T_{\max }-T_{\min }\right) /\left(T_{\max }+T_{\min }\right)$, where $T_{\max }$ and $T_{\min }$ are the maximum and minimum values of the trace over a certain distance record (in our case, a window of $40 \mathrm{~m}$ ).

and the sidebands transfer back some power to the central laser line. In the $\phi \mathrm{OTDR}$ trace, this manifests as a recovery of the contrast in the recorded interferences. Over long distances, this reversible power-transfer process between the central peak and the sidebands can switch several times, leading to an oscillatory pattern in the visibility of the interferences measured along the fiber.

Further insight into this explanation can be obtained by looking at the output spectra recorded in the OSA. Figure 3 shows a plot of the optical power in the peak and the integrated power contained in the MI sidebands as recorded by the OSA at the end of the fiber for different input powers. The results have been normalized by the total power recorded and by subtracting the DC component given by the ASE of the EDFA. We can observe a similar effect to the evolution along the fiber recorded in Fig. 2. For powers below $400 \mathrm{~mW}$, the spectrum recorded at the fiber output shows that $>90 \%$ of the power is contained in the central wavelength. Therefore, the visibility of the interference signal is expected to remain high all along the fiber. With increasing input pulse powers, more power is contained in the sidebands until a minimum is reached. This minimum power recorded in the central peak is also coincident with a minimum contrast of the interferences recorded in the far end of the $\phi \mathrm{OTDR}$ trace. The inset figures of Fig. 3 show the output spectra for (a) 24.5, (b) 27.8, and (c) $28.8 \mathrm{dBm}$ of input peak powers. A clear peak power decrease and sidebands power increase is observed between the spectra recorded at 24.5 and $27.8 \mathrm{dBm}$ input power, and then a smaller increase of peak power and decrease of sidebands power can also be observed between the spectra collected at 27.8 and $28.8 \mathrm{dBm}$ input peak power. This 


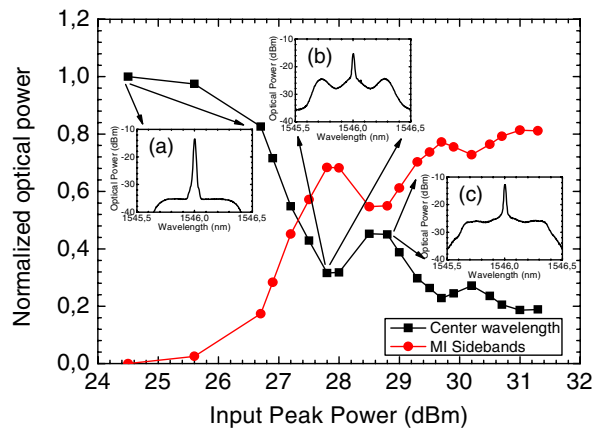

Fig. 3. (Color online) Normalized optical power of the peak and sidebands at the end of the fiber for different input pump powers. Inset figures: spectrum for input pump powers of (a) 24.5, (b) 27.8, and (c) $28.8 \mathrm{dBm}$.

corresponds also to an increase of the interference visibility at the far end of the fiber. A clear signature of the FPU recurrence can therefore be also recorded with this simple graph.

To numerically corroborate this phenomenon, we also simulated the light propagation along our fiber under test (FUT) by numerically solving the nonlinear Schrödinger equation for the scalar electric field envelope $A(z, t)$ :

$$
\frac{\partial A}{\partial z}+\frac{i \beta_{2}}{2} \frac{\partial^{2} A}{\partial t^{2}}-\frac{\beta_{3}}{6} \frac{\partial^{3} A}{\partial t^{3}}+\frac{\alpha}{2} A=i \gamma|A|^{2} A
$$

Equation (1) was solved using a split-step Fourier algorithm [4] with adaptive step size. The fiber parameters used in the simulation were those of a typical SMF-28 at the wavelength of our laser: attenuation coefficient $\alpha=0.2 \mathrm{~dB} / \mathrm{km}, \quad$ nonlinear coefficient $\gamma=1.3 \mathrm{~W}^{-1} \cdot \mathrm{km}^{-1}$, group velocity dispersion coefficient $\beta_{2}=-22.8 \mathrm{ps}^{2} \mathrm{~km}^{-1}$ (corresponding to a dispersion coefficient of $D=18 \mathrm{ps} \mathrm{nm}{ }^{-1} \cdot \mathrm{km}^{-1}$ ) and third-order dispersion coefficient $\beta_{3}=-0.1 \mathrm{ps}^{3} \cdot \mathrm{km}^{-1}$. The pulse peak power was consistent with the one recorded from the experiments. The input noise was considered flat.

The results obtained in the simulation showed an excellent agreement with the explanation given above. As an example, we plot in Fig. 2 the fraction of power contained in the center wavelength as a function of the fiber length (for the same input power conditions as those recorded for Fig. 2). As is visible, the positions where the contrast of the trace is higher correspond to positions where the model predicts a high fraction of power in the central wavelength. On the contrary, the positions where the $\phi$ OTDR trace provides the smallest contrast correspond to positions where the power in the central peak is minimal. It is worth saying that in the fading points, the peak power in the central line estimated from simulation reached $\sim 10 \%$ of the spectral power while the measured visibility in these positions was $3 \%-5 \%$. We believe that this discrepancy may be caused by a nonideal visibility of the experimental input pulses and also by a nonflat distribution of the input noise. We have verified by simulation that introducing a higher input noise close to the central peak leads to a deeper depletion of the central line. The actual spectra given by the simulation along the fiber length can be seen in Fig. $\underline{4}$ (same power

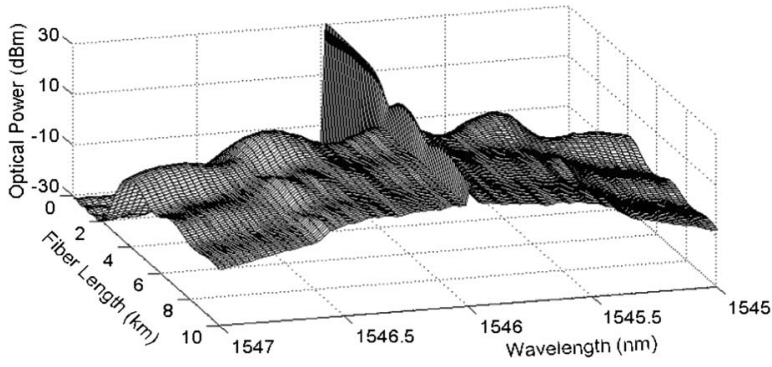

Fig. 4. Simulation of the input pulse spectrum evolution along the fiber using the parameters of the main figure of Fig. 2.

settings as Fig. 2). We can check in the simulations that the positions where the power in the peak shows a dip correspond to positions where the power in the sidebands is maximized, and vice versa, again evidencing FPU recurrence. The theoretical model therefore shows good agreement with the evolution of the interference signal recorded by the experimental results, with the spectral measurements recorded at the fiber end and our own qualitative explanation of the obtained results.

In conclusion, we have provided an in-depth explanation of the effects of MI on $\phi$ OTDR traces. We have shown experimentally that MI leads to a positiondependent fading of the $\phi$ OTDR trace associated with a loss of power at the narrow laser wavelength in favor of the MI sidebands, and therefore with a loss of sensitivity in these positions. The fading is position dependent due to the reversible power transfer between the main input laser line and the MI sidebands (FPU recurrence). Although this effect can be considered detrimental in the context of $\phi \mathrm{OTDR}$, we believe that it can clearly be a convenient and highly visual way of evidencing the FPU recurrence in optical fibers.

We acknowledge funding from the European Research Council through Starting Grant U-FINE (Grant No. 307441), the Spanish "Plan Nacional de I+D+i" through projects TEC2012-37958-C02-01 and TEC201237958-C02-02, the regional program FACTOTEM2 funded by the Comunidad de Madrid, and the INTERREG SUDOE program ECOAL-MGT. HFM acknowledges funding from the Portuguese Fundação para a Ciência e Tecnologia (FCT) for providing his Ph.D. scholarship, SFRH/BD/76991/2011.

\section{References}

1. Y. L. Lu, T. Zhu, L. A. Chen, and X. Y. Bao, J. Lightwave Technol. 28, 3243 (2010).

2. J. C. Juarez, E. W. Maier, K. N. Choi, and H. F. Taylor, J. Lightwave Technol. 23, 2081 (2005).

3. H. Izumita, Y. Koyamada, S. Furukawa, and I. Sankawa, J. Lightwave Technol. 12, 1230 (1994).

4. G. P. Agrawal, Nonlinear Fiber Optics, 3rd ed. (Academic, 2001).

5. G. Van Simaeys, P. Emplit, and M. Haelterman, J. Opt. Soc. Am. B 19, 477 (2002).

6. G. Van Simaeys, P. Emplit, and M. Haelterman, Phys. Rev. Lett. 87, 033902 (2001).

7. D. Alasia, M. G. Herraez, L. Abrardi, S. M. Lopez, and L. Thevenaz, Proc. Soc. Photo-Opt. Instrum. Eng. 5855, 587 (2005). 